\title{
Spatial and Dynamic Analysis of Regional Sustainable Development Using Geographic Information System and Relative Carrying Capacity of Resources*
}

\author{
Qi Wang ${ }^{1 \#}$, Hua Tang ${ }^{1}$, Jun $\mathrm{Li}^{1}$, Haihu Ma ${ }^{1}$, Tianxing Cheng ${ }^{2}$, Xiaodan Wang ${ }^{3}$ \\ ${ }^{1}$ College of Life and Environmental Science, Wenzhou University, Wenzhou, China; ${ }^{2}$ College of Chemistry and Materials Engineer- \\ ing, Wenzhou University, Wenzhou, China; ${ }^{3}$ School of Chemistry and Chemical Engineering, Southwest University, Chongqing, \\ China Email: \#victor527@126.com,wangqi@wzu.edu.cn
}

Received December $13^{\text {th }}, 2010$; revised January $24^{\text {th }}, 2011$; accepted January $31^{\text {st }}, 2011$.

\begin{abstract}
Relative carrying capacity of resources is an index to measure sustainable development through carrying capacity. Case studies of eleven cities in Zhejiang (Hangzhou, Ningbo, Wenzhou, Jiaxing, Huzhou, Shaoxing, Jinhua, Quzhou, Zhoushan, Taizhou and Lishui) illustrated regional sustainable development approach. In this study, to provide insight into spatial and dynamic analysis of region sustainable development, we calculated the relative carrying capacity of land resources and economical resources and synthetical carrying capacity of resources in different cities in Zhejiang, and geographic information system was carried out. The results showed that all cities but Hangzhou and Ningbo were ecologically sustainable, and relative carrying capacity of land resources in northern and eastern Zhejiang was larger than those in southern and western Zhejiang. The sampling years of Wenzhou, Hangzhou and Ningbo contribution rates of land resource to synthetic carrying capacity were grouped into three stages, and there were two milestones trends and changes in 1996 and 2004, respectively. This study demonstrated that geographic information system and relative carrying capacity of resources are effective for assessment of region sustainable development, and provide policy guidelines for decision-making.
\end{abstract}

Keywords: Geographic Information System, Arcgis, Relative Carrying Capacity of Resources, Regional Sustainable Development, Contribution Rate

\section{Introduction}

Sustainable development has been widely recognized as an effective tool for harmonizing human society and nature. The main research and practice of sustainable development concept are focused on efficient use of the human and economical resources. Assessment of sustainable development for effective regional management is becoming concern, which included Relative Carrying Capacity of Resources (RCCR) [1], Ecological Footprint (EF) [2,3], Index of Sustainable Economic Welfare (ISEW) [4], Environmental Decision Support System (EDSS) [5], and Genuine Progress Indicator(GPI) [6] .

Wang proposed that carrying capacity of natural reso-

*The authors claim that the paper was partly published in Proceeding of International Conference on Environmental Science and Information Application Technology in 2009 and exchanged in this conference. urces was much lower than the carrying capacity of economy resources of Wenzhou and had been the state of overload from 1992 to 2004 [1]. Ivan and Anna explored the determinants of the ecological footprint of commuting municipal variability by using population density, accessibility, average household income, and job ratio. Nguyen and Yamamoto reported the estimated total ecological footprint of the world using the new method implied more serious problems associated with over consumption than using results from the original ecological footprint method.

Geographic information system (GIS) is a useful and effective tool for spatial features in various fields, particularly in environmental science. In recent years, it has been widely studied for geographical distribution and spatial analysis including particulate waste distribution 
[7], ecological connectivity [8], acute symptoms [9], paramphistomosis in sheep [10], verotoxin-producing Escherichia coli [11], etracapsuloides bryosalmonae infected fishes [12], international epidemiology of lung cancer [13] variance of intraseasonal variations [14], PBDEs in human breast milk [15], amino acid mutations [16], and Colletotrichum lindemuthianum [17].

Yokoi and Satomura revealed remarkable differences ill the geographical distribution, of variance between two types of intraseasonal variations in daily-mean radar reflectivity data ill the western part of the Indochina Peninsula [14]. Sudaryanto et al. proposed that concentrations of PBDEs were relatively uniform and the levels were in the same order as those in Japan and some European countries, but were one or two order lower than North America by using geographical distribution [15].

RCCR is essential for carrying capacity in order to assess regional sustainable development. At present, quantitative studies on the spatial analysis of regional sustainable development based on GIS and RCCR in a given region are still rare. With the rapid economic development, Zhejiang has faced with severe conflicts between limited natural resources and increasing resource requirements. Therefore, Zhejiang has become a great challenge for sustainable development over the last decades.

The present study based on [18] aimed to evaluate spatial and dynamic difference of regional sustainable development based on geographic information system and relative carrying capacity of resources in Zhejiang.

\section{Studied Area}

Zhejiang is located in the southern part of the Yangtze River Delta on the southeast coast of China. It covers a total land area of 101800 square kilometers. Hills and mountains account for 70.4 percent of the total area in the province. The permanent population of the province reached 51.2 million by the end of 2008, an increase of $1.19 \%$ over the previous year. It reserves of stone coal, alunite, pyrophyllite, and tuff rank the first in China and the reserves of fluorite rank the second. In addition, rich deposits of oil and natural gas in the continental shelf are awaiting exploitation. There are 11 cities under the direct jurisdiction of Zhejiang provincial government, including Hangzhou, Ningbo, Wenzhou, Jiaxing, Huzhou, Shaoxing, Jinhua, Quzhou, Zhoushan, Taizhou and Lishui.

\section{Methodology}

\subsection{Relative Carrying Capacity of Land Resources}

Relative carrying capacity of land resources calculation equation is as follows:

$$
C_{r l}=I_{l} Q_{l}
$$

where $C_{r l}$ is relative carrying capacity of land resources, $Q_{l}$ is the study area cultivated land, $I_{l}$ is the study area nature resource carrying capacity index, $I_{l}=$ $Q_{p 0} / Q_{10}$, and $Q_{p 0}$ is reference region population in the country, $Q_{10}$ is reference region cultivated land in the country.

\subsection{Relative Carrying Capacity of Economy Resources}

Relative carrying capacity of economy resources calculation equation is as follows:

$$
C_{r e}=I_{e} Q_{e} .
$$

where $C_{r e}$ is relative carrying capacity of economy resources, $Q_{e}$ is the study area economy, $I_{e}$ is the study area economy carrying capacity index, $I_{e}=Q_{p 0} / Q_{e 0}$, and $Q_{p 0}$ is reference region population in the country, $Q_{e 0}$ is reference region economy in the country.

\subsection{Synthetical Carrying Capacity of Resources}

Synthetical carrying capacity of resources calculation equation is as follows:

$$
C_{s}=W_{1} C_{r l}+W_{2} C_{r e} .
$$

where $C_{s}$ is synthetical carrying capacity of resources, $W_{1}$ is the weight of $C_{r l}$, and $W_{2}$ is the weight of $C_{r e}$. Here $W_{1}$ is 0.7 and $W_{2}$ is 0.3 according to actual nature resources conditions in Zhejiang[1].

\subsection{Contribution Rate of Land Resource to Synthetic Carrying Capacity}

Contribution rate of land resource to synthetic carrying capacity calculation equation is as follows:

$$
\mathrm{Y}_{l}=\frac{C_{r l} \times 0.7}{C_{s}} \times 100 \% \text {. }
$$

where $Y_{l}$ is contribution rate of land resource to synthetic carrying capacity.

\subsection{Geographic Information System}

A GIS can integrate hardware, software, and data for capturing, managing, analyzing, and displaying all forms of geographically referenced information and can show features and feature relationships. The assessment and calculation data of relative carrying capacity of resources used in the analysis were drawn from Zhejiang statistical yearbooks in 2008. The spatial map analysis was performed by the ArcGIS 9.2 for Windows (Environmental al Systems Research Institute, Inc., USA) software packages.

Firstly, display Zhejiang province map features and 
create selection layers. Secondly, add data such as calculated relative carrying capacity of land resources to Zhejiang province map. Thirdly, edit geographic data, and select geographic features. Fourthly, create a summary chart. Finally, lay out and print the maps of relative carrying capacity. The units of relative carrying capacity of resources are all ten thousand persons from Figure 1 to Figure 10 except Figure 6.

The flow chart of the GIS-based method for spatial analysis of regional sustainable development is shown in Figure 1.

\section{Results and Discussion}

Over load population relative to carrying capacity in Zhejiang is shown in Figure 2.

Number of over load population is positive, which means ecologically unsustainable. On the contrary, number of over load population is negative, which means ecologically sustainable. Figure 2 showed that sustainable development level of Ningbo was the best, while that of Wenzhou was the worst because its over load population relative to carrying capacity is the largest and all cities but Hangzhou and Ningbo were ecologically sustainable. The sustainable development level order of over load population relative to carrying capacity in Zhejiang was as follows: Ningbo, Hangzhou, Jiaxing, Shaoxing, Huzhou, Zhoushan, Quzhou, Jinhua, Lishui, Taizhou and Wenzhou. Spatial sustainable development level in northern Zhejiang is better than those in southern Zhejiang.

Spatial map of relative carrying capacity of land resources in Zhejiang is shown in Figure 3.

Figure 3 showed that relative carrying capacity of land resources in northern and eastern Zhejiang was larger than those in southern and western Zhejiang, indicating resources quality in northern and eastern Zhejiang

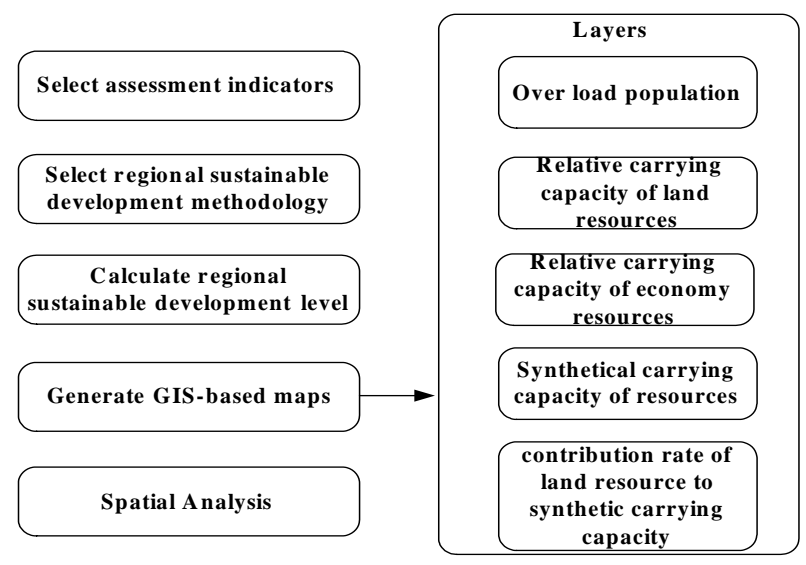

Figure 1. Flow chart of the GIS-based method for spatial analysis of regional sustainable development.

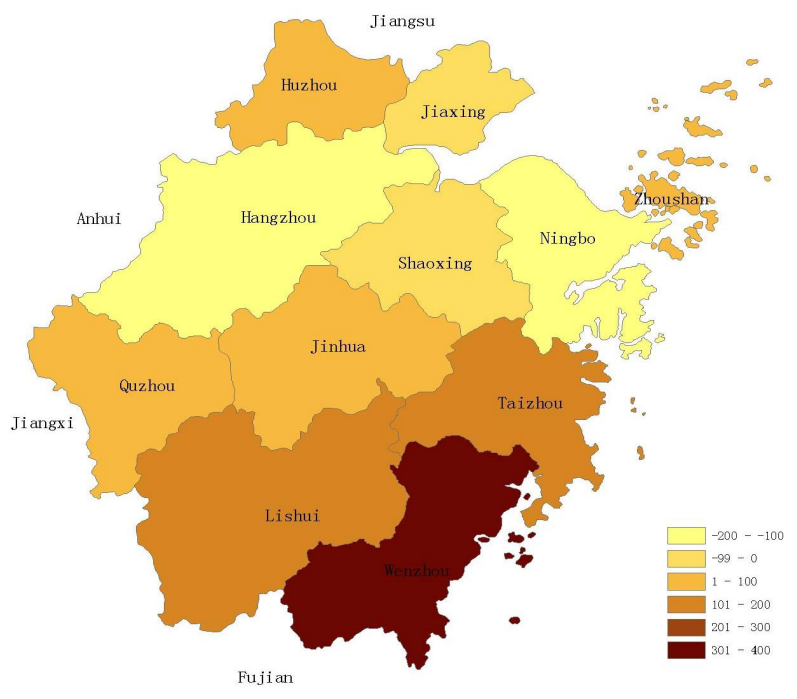

Figure 2. Spatial map of over load population relative to carrying capacity in Zhejiang.

better than those in southern and western Zhejiang. Spatial map of relative carrying capacity of economy resources is shown in Figure 4.

Figure 4 showed that relative carrying capacity of economy resources of Hangzhou, Ningbo and Wenzhou were larger than other those cities in Zhejiang, indicating economy development level in Hangzhou, Ningbo and Wenzhou better than those of other cities in Zhejiang.

Spatial map of synthetical carrying capacity of resources in Zhejiang is presented in Figure 5.

As can be seen in Figure 5, Zhoushan is located in the northeast of Zhejiang. It's an important gateway of inland place to the outside world and the juncture of water arteries linking south and north China with the Yangtse

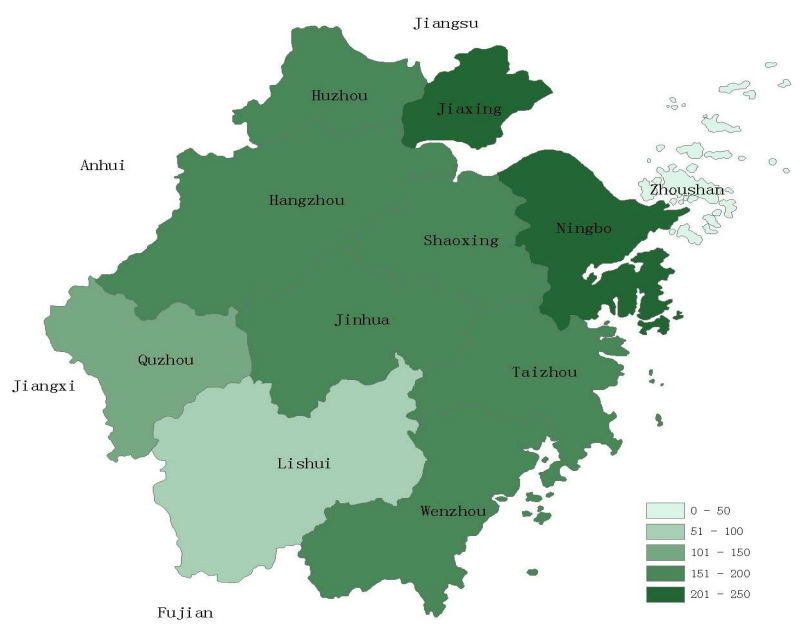

Figure 3. Spatial map of relative carrying capacity of land resources in Zhejiang. 


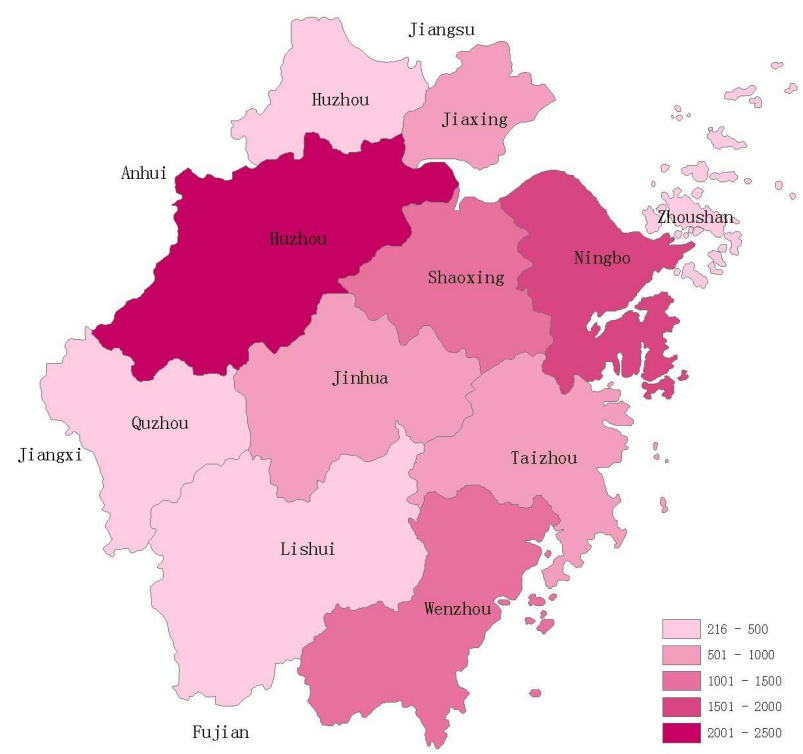

Figure 4. Spatial map of relative carrying capacity of economy resources in Zhejiang.

River. Quzhou is located in the west of Zhejiang, and is usually described as "the Artery of Four Provinces, the Western Gate of Zhejiang" which has rich mineral resources. Lishui is located in the combination of South Zhejiang and North Fujian mountainous regions. Lishui is very rich in resources and specialties with the total amount of five major natural resources ranking top among the province, i.e. forest, waterpower, agricultural and byproducts, mineral resources, wild animals and plants. Figure 5 showed that Zhoushan, Quzhou and

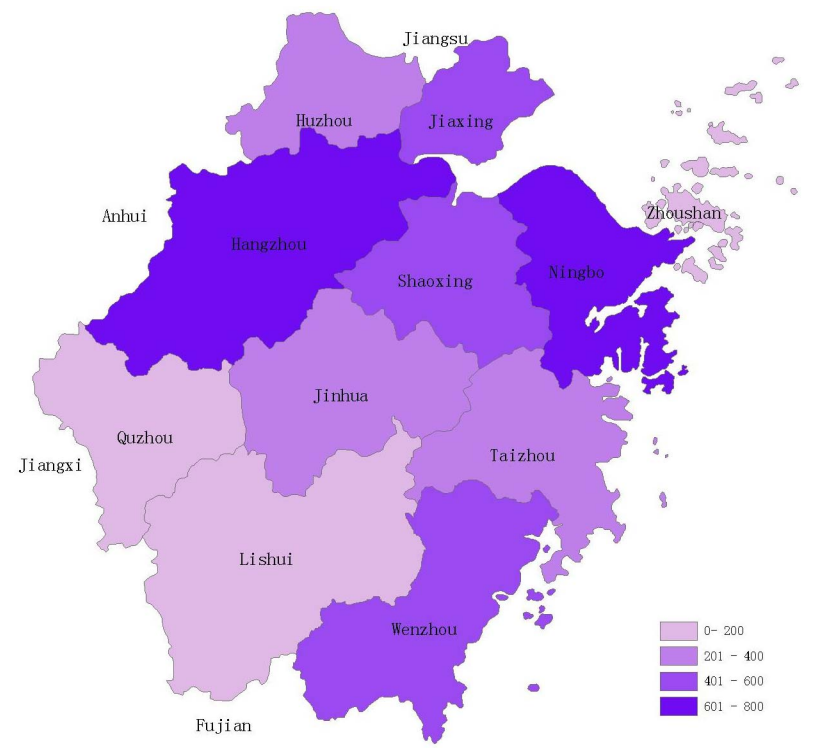

Figure 5. Spatial map of synthetical carrying capacity of resources in Zhejiang.
Lishui have the lower synthetical carrying capacity of resources in Zhejiang, whereas Hangzhou and Ningbo have the higher synthetical carrying capacity of resources in Zhejiang. The synthetical carrying capacity of resources in Zhejiang from the highest to the lowest was as follows: Hangzhou, Ningbo, Wenzhou, Shaoxing, Jiaxing, Taizhou, Jinhua, Huzhou, Quzhou, Lishui and Zhoushan. Although Wenzhou had relatively larger synthetical carrying capacity of resources, over load population was serious and sustainable development level of Wenzhou was the worst.

Spatial map of contribution rate of land resource to synthetic carrying capacity in Zhejiang is presented in Figure 6.

Figure 6 showed that contribution rates of land resource to synthetic carrying capacity in western Zhejiang were larger than those in eastern Zhejiang except Hangzhou. Slower economy, larger contribution rates of land resource to synthetic carrying capacity. Therefore policies of nature resource become a crucial factor for growth of sustainable development in Zhejiang.

Dynamic analysis of regional sustainable development using geographic information system and relative resource carrying capacity was developed for the cases of Hangzhou, Wenzhou and Ningbo. Dynamic plot of over load population relative to carrying capacity in Zhejiang is presented in Figure 7.

Figure 7 showed that the number of over load population declined rapidly from 1993 to 2004, and increased rapidly from 2004 to 2005, and keep calm 2005 to 2007

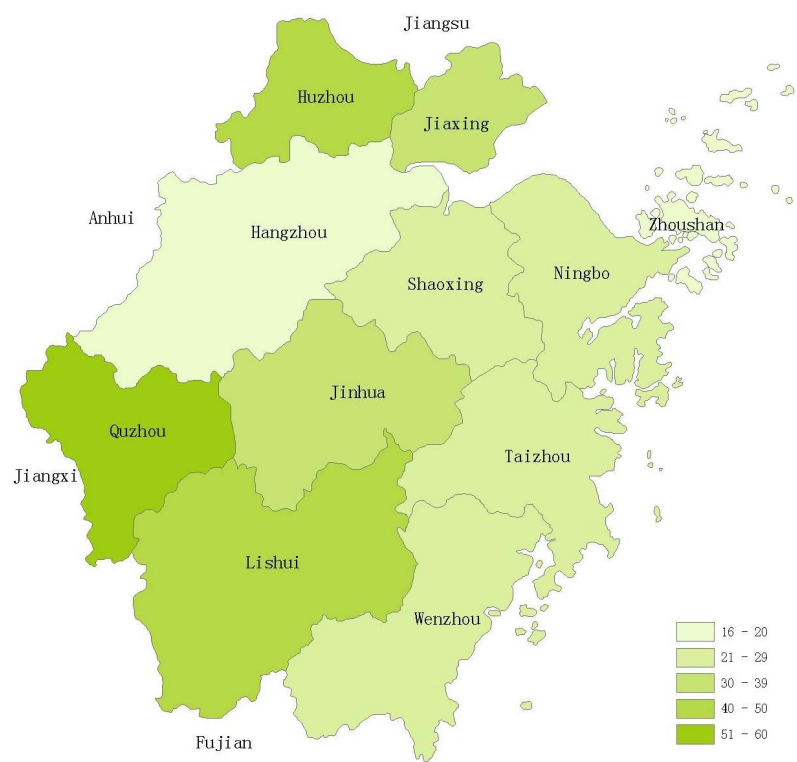

Figure 6. Spatial map of contribution rates of land resource to synthetic carrying capacity in Zhejiang. 


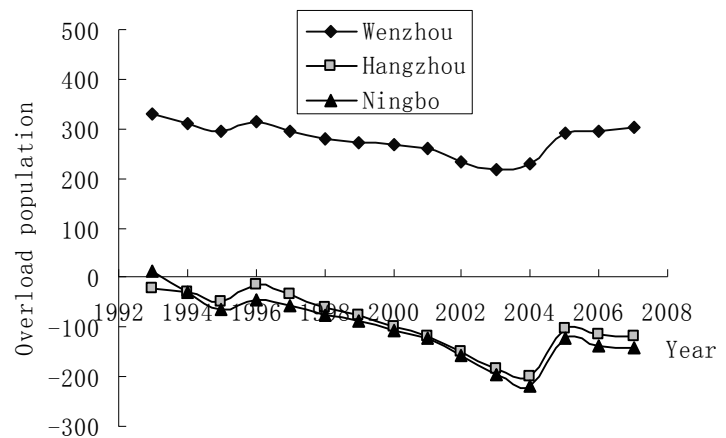

Figure 7. Dynamic plot of over load population relative to carrying capacity in Zhejiang.

in Zhejiang such as Hangzhou, Wenzhou and Ningbo. The three cities of Zhejiang province such as Wenzhou, Hangzhou and Ningbo have experienced significant structural changes due to the incessant growth in the number of urban population.

The over load population of Wenzhou varied from 2 196 thousand person to 3291 thousand person during 1993 to 2007 with an average value of 2793 thousand person. The over load population of Hangzhou and Ningbo varied from -127 and 138 thousand person to -2012 and -2 189 thousand person during 1993 to 2007 with an average value of -912 and -1033 thousand person, respectively, indicating Hangzhou and Ningbo non over load level of sustainable development from 1993 to 2007.

Dynamic plot of relative carrying capacity of land resources in Zhejiang is presented in Figure 8.

Figure 8 showed that relative carrying capacity of land resources in Zhejiang remained essentially constant from 1993 to 1995 , while it increased with steady steps from 1996 to 2007. The order of relative carrying capacity of land resources from higher to lower as followed: Ningbo, Hangzhou and Wenzhou. The prevention and control land resource of Wenzhou was still severe.

Dynamic plot of relative carrying capacity of economy

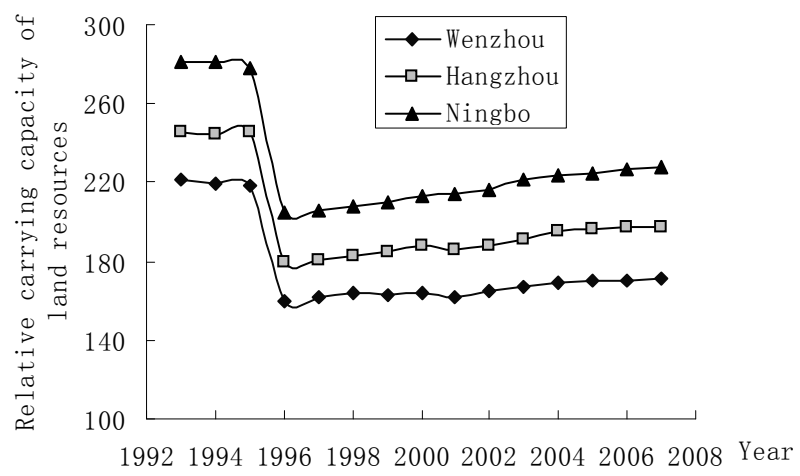

Figure 8. Dynamic plot of relative carrying capacity of land resources in Zhejiang. resources in Zhejiang is presented in Figure 9.

Figure 9 showed that relative carrying capacity of economy resources increased steadily from 1993 to 2004, whereas it remained essentially constant from 2005 to 2007.

The order of relative carrying capacity of economy resources from higher to lower as followed: Hangzhou, Ningbo and Wenzhou.

Dynamic plot of synthetical carrying capacity of resources in Zhejiang is presented in Figure 10.

Figure 10 showed that synthetical carrying capacity of resources was increased steadily from 1993 to 2004, whereas remained essentially constant from 2005 to 2007.

The sampling years of Wenzhou, Hangzhou and Ningbo synthetical carrying capacity of resources were grouped into two stages. The order of synthetical carrying capacity of resources from higher to lower as followed: Hangzhou, Ningbo and Wenzhou.

Synthetical carrying capacity of resources in Zhejiang increased with increasing relative carrying capacity of economy resources in Zhejiang, and Synthetical carrying capacity of resources declined with declining relative carrying capacity of economy resources.

Dynamic plot of contribution rates of land resource to synthetic carrying capacity in Zhejiang is presented in Figure 11.

Figure 11 showed that sampling years of Wenzhou, Hangzhou and Ningbo contribution rates of land resource to synthetic carrying capacity were grouped into three stages, and there were two milestones in the contribution rates of land resource to synthetic carrying capacity temporal trends and changes, which year were 1996 and 2004. The contribution rates of land resource to synthetic carrying capacity increased suddenly from 1993 to 1996 , and increased steadily from 1996 to 2004, whereas it remained essentially constant from 2005 to 2007.

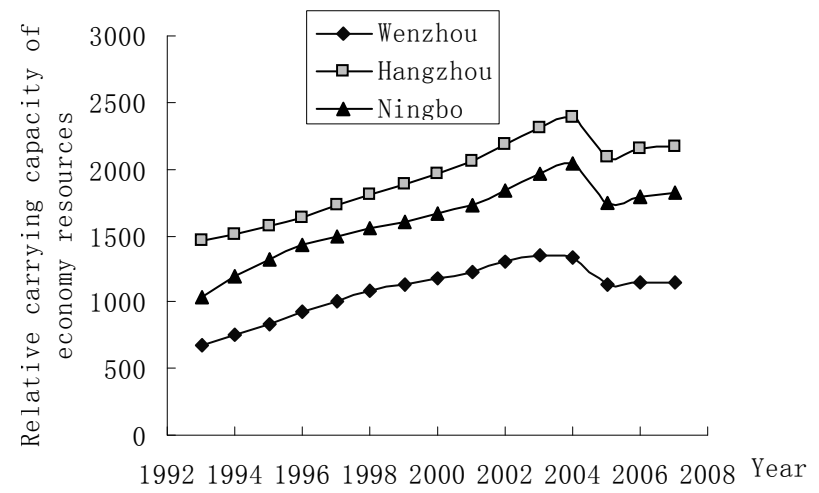

Figure 9. Dynamic plot of relative carrying capacity of economy resources in Zhejiang. 


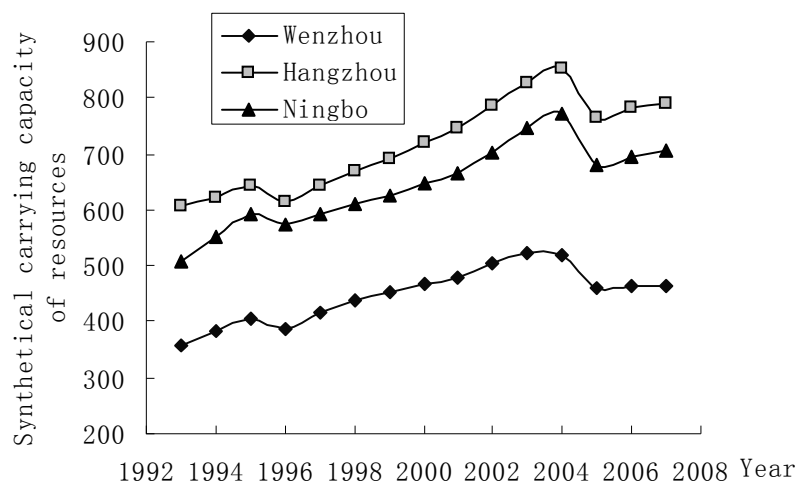

Figure 10. Dynamic plot of synthetical carrying capacity of resources in Zhejiang.

The order of synthetical carrying capacity of resources from higher to lower as followed: Wenzhou, Ningbo and Hangzhou.

In order to achieve "double-win" of economy and environment, the local government should take into considerations in developing circular economy including construction of eco industrial park (EIP).

Thus, the city's policies of sustainable development in the future should aim at emphasis on strengthening population and nature resources management, upgrading industrial structure, and raising the utilization efficiency of resources based on different regional situations.

\section{Conclusions}

Relative carrying capacity of land resources in northern and eastern Zhejiang was larger than those in southern and western Zhejiang. Zhoushan, Quzhou and Lishui have the lower synthetical carrying capacity of resources in Zhejiang, whereas Hangzhou and Ningbo have the higher synthetical carrying capacity of resources in Zhejiang. The contribution rates of land resource to synthetic carrying capacity in western Zhejiang were more

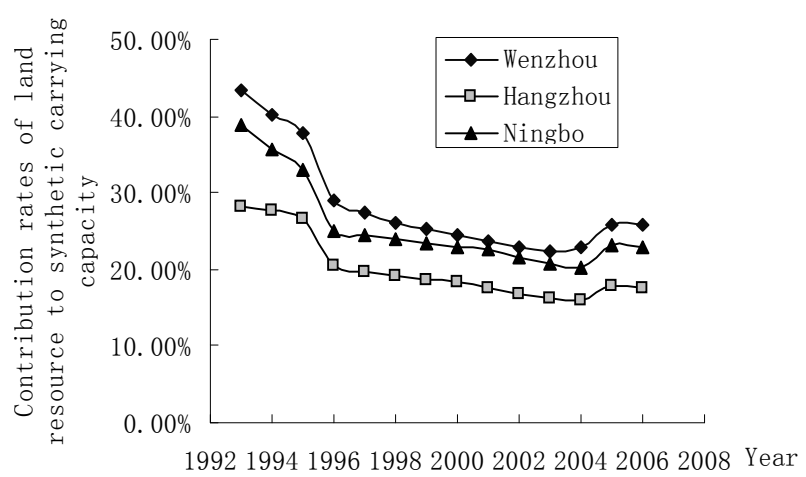

Figure 11. Dynamic plot of contribution rates of land resource to synthetic carrying capacity in Zhejiang. than those in eastern Zhejiang except Hangzhou.

Hangzhou and Ningbo were not over load level of Sustainable development, whereas Wenzhou was seriously over load of that from 1993 to 2007 . Synthetical carrying capacity of resources increased with increasing relative carrying capacity of economy resources in Zhejiang such as Wenzhou, Hangzhou and Ningbo and vice versa.

The geographic information system and regional relative carrying capacity of resources are useful tools to measure urban sustainable development and provide policy guidelines for decision-making.

\section{Acknowledgements}

This work was supported in part by a grant from Wenzhou Major soft science Bidding Notices Foundation (R20060004), the grant from Wenzhou Soft Science Project (R20100119).

\section{REFERENCES}

[1] Q. Wang, "Dynamics Analysis on the Relative Carrying Capacity of Resources of Wenzhou," Proceeding Chinese Control Conference (2008CCC), IEEE Press, July 2008, pp. 738-742 (in Chinese). doi:10.1109/CHICC.2008.4604990

[2] I. Muniz and A. Galindo, "Urban Form and the Ecological Footprint of Commuting. The Case of Barcelona," Ecological Economics, Vol. 55, No. 4, 2005, pp. 499-514. doi:10.1016/i.ecolecon.2004.12.008

[3] H. X. Nguyen and R. Yamamoto, "Modification of Ecological Footprint Evaluation Method to Include Non-Renewable Resource Consumption Using Thermodynamic Approach," Resources Conservation and Recycling, Vol. 51, No. 4, 2007, pp. 870-884. doi:10.1016/j.resconrec.2007.01.004

[4] M. Mundayand and A. Roberts, "Developing Approaches to Measuring and Monitoring Sustainable Development in Wales: A Review," Regional Studies, Vol. 40, No. 5, 2006, pp. 535-554. doi:10.1080/00343400600757726

[5] G. H. Huang, X. S. Qin, W. Sun, X. H. Nie and Y. P. Li, "An Optimisation-Based Environmental Decision Support System for Sustainable Development in a Rural Area in China," Civil Engineering and Environmental Systems, Vol. 26, No. 1, 2009, pp. 65-83. doi: $10.1080 / 10286600802003732$

[6] N. Hanley, I. Moffatt, R. Faichney and M. Wilson, "Measuring Sustainability: A Time Series of Alternative Indicators for Scotland," Ecological Economics, Vol. 28, No. 1, 1999, pp. 55-73. doi:10.1016/S0921-8009(98)00027-5

[7] O. M. Perez, T. C. Telfer, M. C. M. Beveridge and L. G. Ross, "Geographical Information Systems (GIS) as a Simple Tool to Aid Modelling of Particulate Waste Distribution at Marine Fish Cage Sites," Estuarine Coastal 
and Shelf Science, Vol. 54, No. 4, 2002, pp. 761-768. doi:10.1006/ecss.2001.0870

[8] J. Marulli and J. M. Mallarach, "A GIS Methodology for Assessing Ecological Connectivity: Application to the Barcelona Metropolitan Area," Landscape and Urban Planning, Vol. 71, No. 2-4, 2005, pp. 243-262. doi:10.1016/S0169-2046(04)00079-9

[9] K. Radon, A. Rosenberger, V. Ehrenstein, M. Hoopmann, I. Basting, H. Todt, J. Reichert, H. Dressel, M. Schmid, R. Suchenwirth and D. Nowak, "Geographical Distribution of Acute Symptoms after a Train Collision Involving Epichlorohydrin Exposure," Environmental Research, Vol. 102, No. 1, 2006, pp. 46-51. doi:10.1016/j.envres.2006.01.010

[10] G. Cringoli, R. Taddei, L. Rinaldi, V. Veneziano, V. Musella, C. Cascone, G. Sibilio and J. B. Malone, "Use of Remote Sensing and Geographical Information Systems to Identify Environmental Features that Influence the Distribution of Paramphistomosis in Sheep from the Southern Italian Apennines," Veterinary Parasitology, Vol. 122, No. 1, 2004, pp. 15-26. doi:10.1016/j.vetpar.2004.03.011

[11] T. Kistemann, S. Zimmer, I. Vagsholm and Y. Andersson, "GIS-Supported Investigation of Human EHEC and Cattle VTEC O157 Infections in Sweden: Geographical Distribution, Spatial Variation and Possible Risk Factors," Epidemiology and Infection, Vol. 132, No. 3, 2004, pp. 495-505. doi:10.1017/S0950268803001729

[12] T. Wahli, D. Bernet, H. Segner and H. Schmidt-Posthaus, "Role of Altitude and Water Temperature as Regulating Factors for the Geographical Distribution of Tetracapsuloides Bryosalmonae Infected Fishes in Switzerland," Journal of Fish Biology, Vol. 73, No. 9, 2008, pp. 2184-2197. doi:10.1111/j.1095-8649.2008.02054.x
[13] D. R. Youlden, S. M. Cramb and P. D. Baade, "The International Epidemiology of Lung Cancer-Geographical Distribution and Secular Trends," Journal of Thoracic Oncology, Vol. 3, No. 8, 2008, pp. 819-831. doi:10.1097/JTO.0b013e31818020eb

[14] S. Yokoi and T. Satomura, "Geographical Distribution of Variance of Intraseasonal Variations in Western Indochina as Revealed from Radar Reflectivity Data," Journal of Climate, Vol. 21, No. 19, 2008, pp. 5154-5161. doi:10.1175/2008JCLI2153.1

[15] A. Sudaryanto, N. Kajiwara, S. Takahashi, Muawanah and S. Tanabe, "Geographical Distribution and Accumulation Features of Pbdes in Human Breast Milk from Indonesia," Environmental Pollution, Vol. 151, No. 1, 2008, pp. 130-138. doi:10.1016/i.envpol.2007.02.016

[16] K. Rungsihirunrat, C. H. Sibley, M. Mungthin and K. Na-Bangchang, "Geographical Distribution of Amino Acid Mutations in Plasmodium Vivax DHFR and DHPS from Malaria Endemic Areas of Thailand," American Journal of Tropical Medicine and Hygiene, Vol. 78, No. 3, 2008, pp. 462-467.

[17] G. A. Bardas, O. Koutita and K. Tzavella-Klonari, "GeoGraphical Distribution, Pathotype Characterization, and Molecular Diversity of Colletotrichum Lindemuthianum in Greece and Resistance of Greek Bean Cultivars," Plant Disease, Vol. 91, No. 11, 2007, pp. 1379-1385. doi:10.1094/PDIS-91-11-1379

[18] Q. Wang, H. H. Ma, Q. Ke, C. H. Wang and X. D. Wang, "Spatial Analysis of Regional Sustainable Development Based on Geographic Information System and Relative Carrying Capacity of Resources," Environmental Science and Information Application Technology. International Conference on ESIAT 2009, Vol. 1, 2009, pp.437-440. 